\title{
Study on Museum Digital Exhibition Mode and Industrialization of Intangible Cultural Heritage
}

\author{
Jian-Song Bai, Jong-Chul Boo, Member, KIMICS
}

\begin{abstract}
Nowadays, most of exhibits are material antiquities while the exhibit of intangible cultural heritage is deficient and the form is less advanced which cannot meet the exhibition needs of digital times. This has resulted in insufficient display of intangible heritage's value which may not facilitate inherit and protection of intangible heritage, no other than protection mechanism of forming healthy industrialization. This article has established digital exhibition mode in museum about intangible cultural heritage by analysis on current museum and using mobile communication and augmented reality techniques. Discuss the mode in the layer integrating creative industry and mobile industry and provide practicable reference project of forming the healthy industrialization mechanism for protecting intangible heritage.
\end{abstract}

Index Terms-protection of intangible heritage, digital exhibition of museum, augmented reality, industrialization

\section{INTRODUCTION}

NOWADAYS, most of museum exhibition tends to exhibit material antiquities. Intangible heritage, an important part of human's heritage, is only exhibited by simple photos and words description or video introduction. The behindhand exhibition mode cannot catch up with the information communication characters in digital times, no other than healthy interaction mechanism of intangible cultural heritage protection and industrialization. This has forced us to think deeply that how to go on museum exhibition in digital times, how the inherit of intangible cultural heritage to adapt the information communication in digital times and whether the interaction mechanism of digital exhibition in museum about intangible cultural heritage and industrialization can be formed.

This study is based on digital media design field and thinks innovatively on the exhibition about intangible cultural heritage. This is based on augmented reality, personal terminal and museum, and integrates the intangible cultural heritage, digital exhibition in museum and mobile communication terminal organically. All of these rely on each other and complement each other and industrialization mechanism with healthy cycle is finally

\footnotetext{
Manuscript received February 12, 2011; revised March 9, 2011; accepted March 23, 2011.

Boo, Jong-Chul is with the Dept. of Multimedia Design, DongSeo University, Busan, 608-737 (E- mail: asiavr@hotmail.com)
}

formed. In a word, this may expand the idea of intangible cultural heritage protection and provide new value of intangible cultural heritage in digital times.

\section{THE STRUCTURE OF CURRENT MAINSTREAMING DIGITAL EXHIBITION MODE}

Various digital techniques have been widely used in exhibition field of various kinds of exhibition halls. The exhibition contents are different and it is difficult to classify according to the manifestations. However, there is a common and important character of all digital exhibitions-human-computer interaction (HCI). Therefore, current digital exhibition modes can be divided into the following types according to the $\mathrm{HCI}$ technique:

Base on transducer. Collect users' interaction information by infrared, pressure or temperature transducer, process them by computer technique, and give feedback according to designed procedure. This type of exhibition mode is applied commonly and is relatively mature and has less demand on the equipment, but the transducer is more prone to failure.

Base on communication technique. It refers to the exhibition mode that using mobile network, local network, blue teeth and other transmission methods to interact between the exhibited information and users' mobile terminal directly and indirectly. This exhibition method is mostly initiated by exhibitions and the system operation is stable. However, this mode requires the user is more skilled with the terminal operation and high demand of calculation speed and communication protocol of mobile terminal.

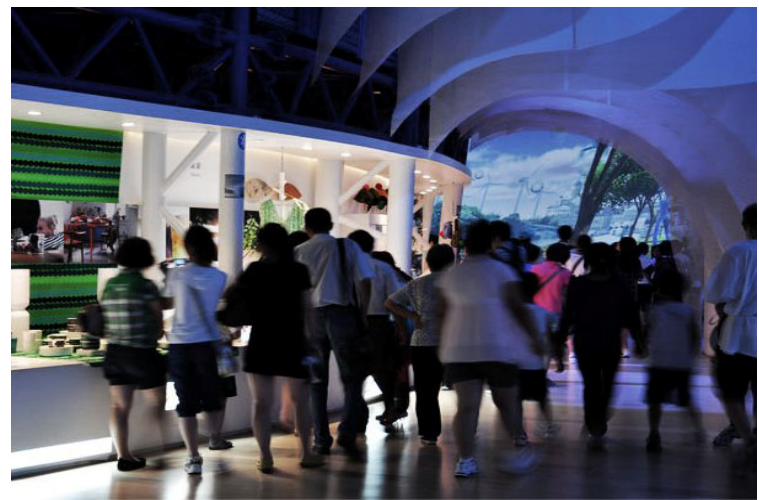

Fig.1. Base on display technique pavilion of footprint, expo 2010 shanghai china. 
Base on display technique. Recover the exhibitions by digital technique and convey information by projection display or optical display methods, such as phantom imaging, air imaging and holographic imaging. This type of mode is characterized by a strong sense of reality of the digital display content and the system is stable because of the closed system, but the requirements of equipments and sites are stricter and the cost of hardware construction and maintenance is higher.

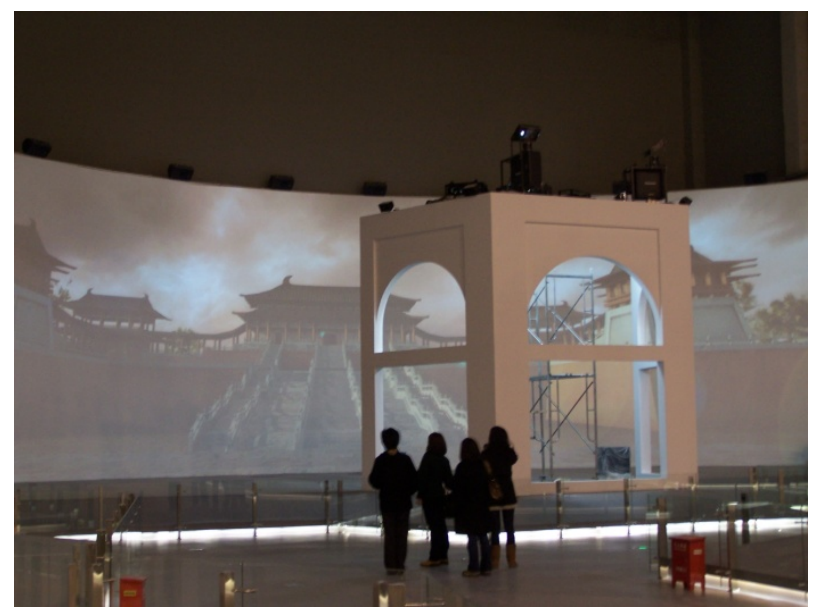

Fig.2. Base on communication finland pavilion, expo 2010 shanghai china.

Base on mechanical control. Viewers operate on the machine designed previously, and the machine will convey the information of digital content. This method is usually used in the display of traditional manual skills. This type of exhibition has a stronger interactive experience sense, because it may feedback the information on the digital monitor through the users' operation on the machine. Therefore, there is no demand on the users' own equipment and the equipment is easy to damage due to high usage rate.



Fig.3. Base on mechanical control jiading bamboo carving museum.
Base on common inquiry machine that is common touch-screen-computer. Users can look up related information by touching the touch-screen. This type is most common and the equipment is mature and stable. It is often used to convey common information, such as the location of pavilion, but it is limited because of its media restrictions.

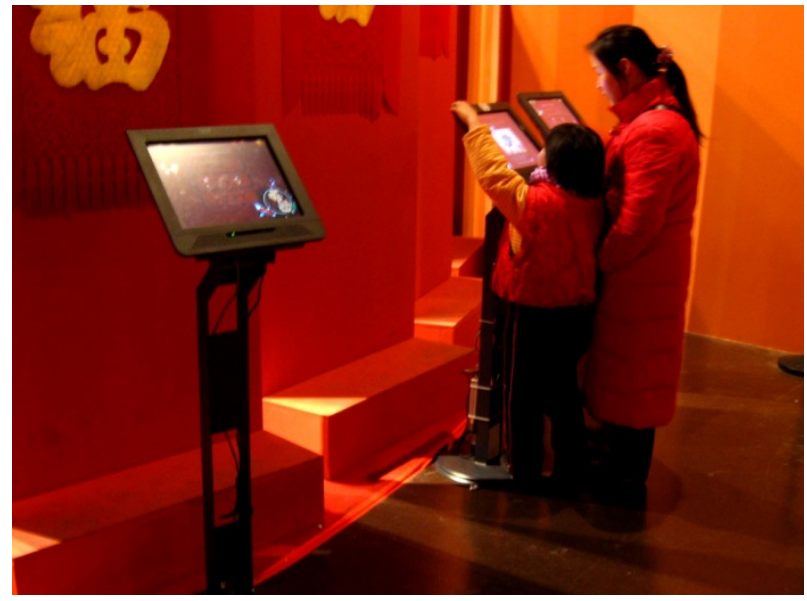

Fig.4. Base on common inquiry machine beijing museum.

\section{ERA OPPORTUNITY OF INTEGRATION OF THREE NETWORKS}

Traditional telephone network begins to develop into the video phone network with visible moving image of opposite side; the traditional one-way broadcast TV communication is growing into two-way interactive TV system, which refers to that users can choose their favorite TV programs according to their hobbies and interests. Another example that cable television network has hundreds of millions of users, if one wants to transmit computer information on cable TV networks, which only need to reform the existing one-way transmission cable into broadband network with the function of two-way communication, thus the concept of "unification of three networks" for integrating computer network, communication network, and TV network is gradually becoming reality. The integration of three networks will win more efficient transmission of various kinds of information and make the network become the real ubiquitous network.

Along with the communication technology grows from $2 \mathrm{G}$ short message service (SMS) to MMS based on 2.5GGPRS technology, WAP, KJAVA and voice valueadded service and to the just commercialized $3 \mathrm{G}$ communication technology not long ago, and from weather forecast to mobile newspaper and innovations of other value-added services and even to the prompting and declared application for important events by government, the mobile phone whose function as information terminals is continually expanded and strengthened has already 
become an important part of public life.

Mobile phone users in China will reach 0.75 billion by 2010, and the handling ability of personal mobile terminal is progressing with each passing day. People go after for the smart phones whose popularity rate is increasing dramatically in a swarm, which provide basic conditions for online service of large computational quantity. To sum up the above terminal characteristics, huge basic number and potential demands of users, it is not difficult for us to see that mobile internet will surely become the communication media of emerging digital content, and while the mobile phone will also certainly become one of the best window for content display.

\section{AUGMENTED REALITY TECHNOLOGY AND MUSEUM EXHIBITION APPLICATION}

In order to preferably satisfy the seamless fitting of real and virtual environment, the emerging technology of augmented reality (Augmented Reality) appears as the times require. Since being proposed by the famous scholar Azumaa and others in 1997, until now the augmented reality that has been widely recognized in the educational circle means that it includes the virtual and real world at the same time, which enable the users not only see the real world, but also can feel the "augmented" effect brought about by computer virtual information. Augmented reality system should have the following features, which namely are virtual and real time interaction, three-dimension rectification.

The applied technology of augmented reality is gradually growing mature and has begun to move towards commercial application. The representatives of TOTAL IMMERSION who is the initiator of augmented reality have already developed the solution for augment reality that is called D'Fusion, which is based on PC platform, of quick operation and very strong compatibility and supports the input and output of high-definition images. The controllers can use D'Fusion to conduct real-time processing of information. At present, this technology is mainly applied in maintenance, marketing and promotion, military simulation, media entertainment, geographical science, etc.

The digitization technology of cultural relics has some applications in digital archives of museum, network exhibition and so on, but the augment reality technology has some incomparable unique advantages that the other technologies do not have in the aspect of digital display design of museum.

a. can improve the exhibition rate and convey efficiency of cultural relics

b. can enrich the information content of exhibition and enjoyment

c. can proceed personalized browsing by breaking the temporal and spatial limit of cultural relics

\section{THE CONCEPT MODE OF MUSEUM EXHIBITION OF INTANGIBLE HERITAGE CONTENT}

In order to enrich the interactive enjoyment of museum exhibition, increase the convey quantity of intangible heritage content in the exhibiting process of cultural relics, the "concept mode of museum exhibition of intangible heritage content" should be established and great effort shall be made to provide museum exhibit design with the references of general meaning through conducting reasonable and effective combination of augmented reality technology and exhibitions and the related display design of intangible heritage content, and again taking the mobile terminal as the medium of information communication.

Being with the center of intangible cultural heritage content, the first part of "mode" proceeds visual design suitable for augmented reality manifestation (e.g. scene mode recovery, figure and event recovery, etc), concentrates on establishing the digital resource repository, and builds identifiable "Marker" index according to the items of exhibition. The second part is the real-time wireless network, which can be divided into wireless LAN and mobile communication network for realizing the invoking of digital resources. The third part conducts identification for the exhibit "Marker" through the camera on mobile terminal, proceeds real-time fitting via combining with the transferred data and video, and then outputs it to the mobile terminal. Mobile terminal includes the customized PDA audio guide provided by the pavilion or the viewers' own $3 \mathrm{G}$ smart phones.

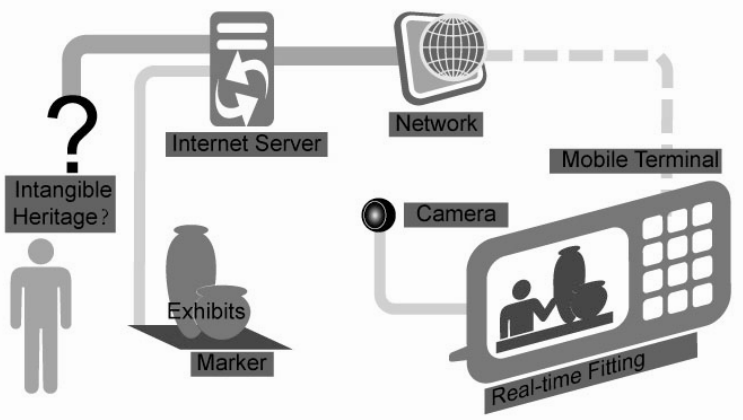

Fig.5. The concept mode of museum exhibition of intangible heritage content.

For further inspecting and verifying the concept mode, this study makes research of practical significance through taking the first museum- - Jiading Bamboo Carving Museum that possesses the "heritage" on the list since the publish of Protection List of National Intangible Cultural Heritage for an example.

The study has conducted the practical application research on the "Sanzhu natural set recovery" and bamboo carving showcase through the investigation and analysis of Jiading Bamboo Carving Museum and combining with "concept mode of museum exhibition of intangible heritage". Firstly, choosing the table screen as one of the bamboo boutique 
forms for digitalization, which will turn to be data file in the database through 3-dimensional mode building, material texture processing, light treatment etc; and then downloading the data file in the database through mobile terminal and outputting it after the fitting with the real image.

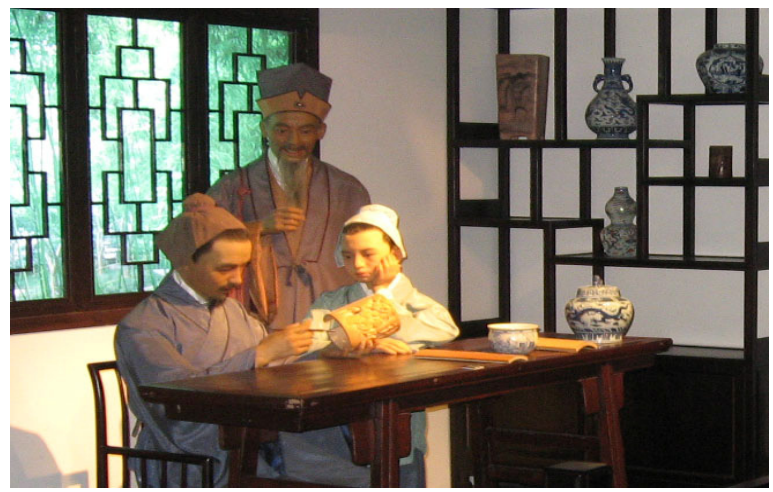

Fig.6. Jiading bamboo carving museum natural set.

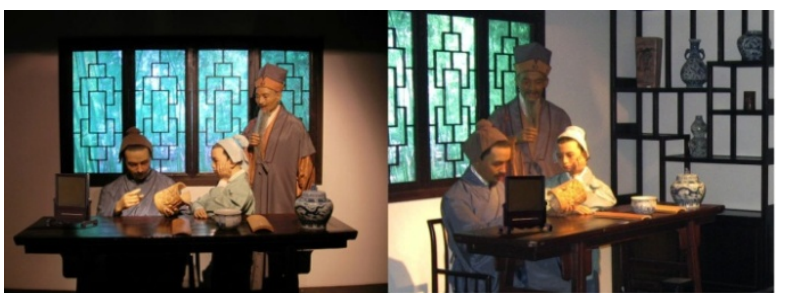

Fig.7. Jiading bamboo carving museum effect of augmented reality.

Scene-type augmented reality means that viewers can not only directly watch the exhibition content, but also can see the real and dynamic location recovery of exhibitions according to the mobile terminal. For examples, the intangible cultural heritage content, vivid representation of historical events, dynamic demonstration of traditional craftsmanship and so on, which are not only suitable for further exhibition and interaction of scene-recovery exhibits, and also suitable for the pavilions relatively lacking of cultural relics or the exhibition items with relatively big space-time bridging.

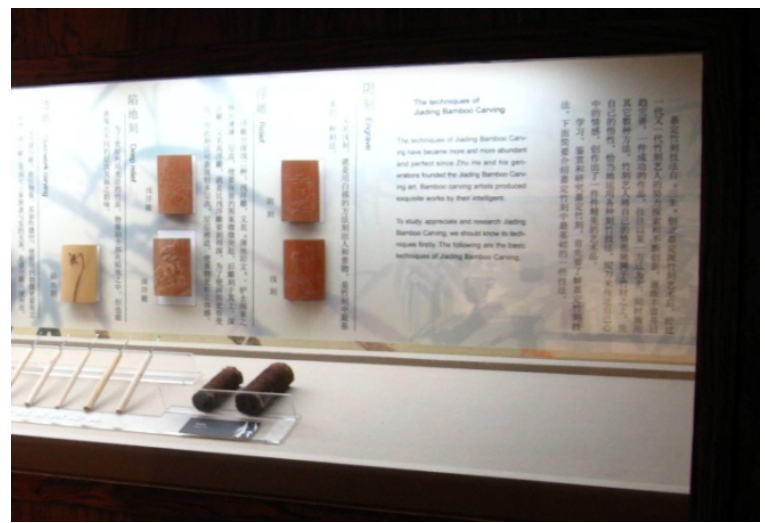

Fig.8. Jiading bamboo carving museum actual location of showcase.



Fig.9. Jiading bamboo carving museum effect of mobile fitting.

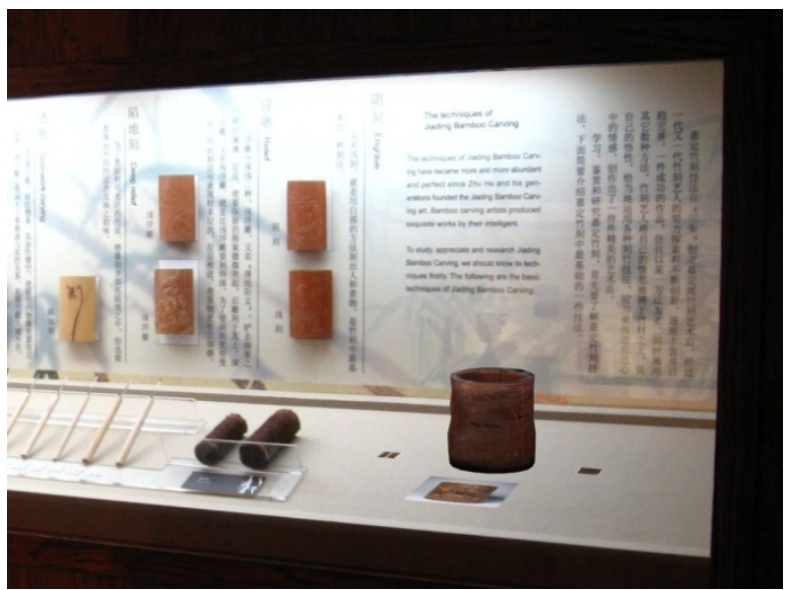

Fig.10. Jiading bamboo carving museum effect of mobile fitting.

For showcase-type augmented reality that can be seen in [Picture-6], viewers can use mobile phones to imagine exhibits from different angles just as the way for watching the physical exhibits. At the same time, there will dynamically appear related type of cultural relics or historical and cultural allusions and so on around the virtual cultural relics, which enable the viewers to further read according to their own needs or even to share their own personal exhibiting content with friends. Such method can be applied for various pavilions, especially suitable for the pavilions with relatively small area of exhibition hall but more exhibits or the ones lacking of explaining personnel and archive pavilions.

The found concept mode of museum exhibition of intangible heritage content in this study can greatly increase the interactive enjoyment of exhibition. Besides, the mode also can provide sound way for inheriting and developing the intangible cultural heritage, and can partially reduce the risks and input cost produced by cultural relic showcases. The transform of visitors from passively viewing the exhibits to actively "explore" them furthermore increases the amount of information and richness of pavilion. 


\section{INDUSTRIALIZATION MODE OF MUSEUM EXHIBITION OF INTANGIBLE HERITAGE}

As public organization, museum has always been the nonprofit-making institution, whose main capital source also comes from the administrative allocation of government that largely leads museum to arrange the exhibition according to the funds. On the other hand, the own nature of intangible cultural heritage that needs numerous capital and time cycle causes exhibits of intangible heritage content being relatively lacking and single. This problem can be solved appropriately through drawing in commercial capital and industrial concepts. Combining with the museum exhibition mode of intangible heritage content, the construct for conducting industrialization mode can be divided into:

In basic level, it includes related scientific research of excavation, sorting out, protection and the reached research findings of intangible heritage by various research institutes. At the present stage, its main funds is from administrative allocation, while the profit redistribution after commercialization can provide valuable supplementary for funds so as to support the research within more wide range.

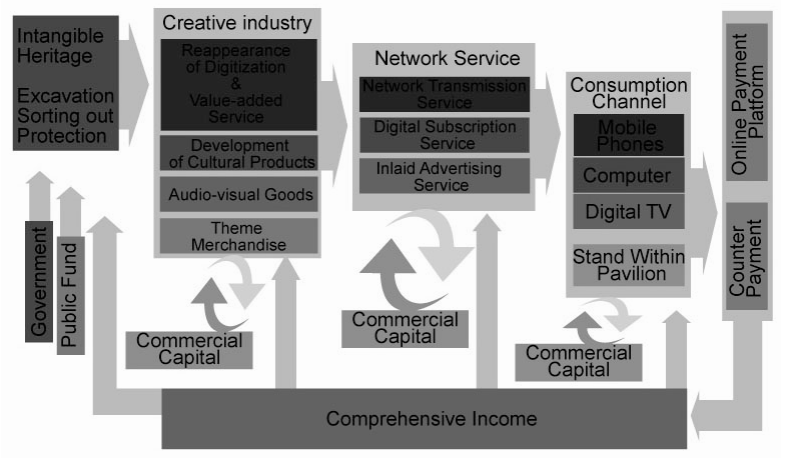

Fig.11. Industrialization mode of museum exhibition of intangible heritage.

Creative industry is also the important innovative part of the industry value of the model, among which includes the development core of intangible heritage contentreappearance of digitization, value-added service, development of cultural products, audio-visual goods and theme merchandise. Proceed reprocessing and recreation of originality on basis of research results so as to make it be more suitable for the cultural demands of consumers. Such part belongs to the development segment of commercialized merchandise, and the related research results of intangible heritage can take part in the cooperation in joint-stock form, thus the organic combination of commerce, industry, knowledge and research can be formed.

Network service is the middle link for innovative products and audiences of intangible heritage content. The subscription service of digital content mainly includes the reappearance of digitization, value-added service, and related cultural products and audio-visual merchandise and so on, which is not only the core of the mode but also the important link for achieving social and commercial value. The inlaid advertising service is the subscription service for digital content, especially for the public welfare and free part, which can provide helpful commercialized supplementary and also can make the museum exhibition of intangible heritage content to realize the maximum public function and ensure the activity for participating in the commercial funds at the same time.

The consumption channel of audience includes mobile phones, personal computer (includes navigation instrument provided by pavilion), digital TV and other major electronic media as well as the physical media of stand within pavilion. The mobile phone among these is the most important way for the communication between the model and the audience, of which the main interactive creation is also additionally realized by the intelligent mobile phone or the smart cable terminal of pavilion. The hardware and network functions of mobile phones have already been basically mature, but the digital content made for it still has huge development space.

The innate payment advantages result from the charging nature of mobile phone, and the auxiliary online payment platform as well as the counter payment, which enable both the users of digital media and the consumers of stand in the pavilion to conveniently accomplish the payment. For achieving more related products of intangible heritage content of more depth and extensiveness, the commercial capital does not hesitate to provide funding support. But considering the commercial risks, the commercial capital will instinctively carry out the supervision and while the research institutes also can provide directional guidance for commercial development.

At the same time, it is not only conducive to preferably protect the freshness of intangible cultural heritage but also to enrich the related digital content service of network. This not only can largely realize the social value of intangible cultural heritage but also can achieve commercial value to some extent, and the both sides supplement and promote for each other, thus a sound and interactive industry circulation mechanism can be truly formed.

\section{CONCLUSIONS}

To some extent, the industrialization of digital exhibition of intangible cultural heritage in museum is indeed a process of media integration. This will establish a more convenient and deeper channel for information transmission and communication between the human's splendid culture heritage and viewers.

Compared with current display modes in pavilions, this mode emphasizes the culture behind the material antiques. 
This concept mode not only focuses on the exhibition of intangible cultural heritage but also display the "code" carried by each exhibit in front of the viewers. This has made it easier for the younger generation to accept and has also enriched and expanded digital industrialization.

Because of the limitation of current technology level and research conditions, some disadvantages still exist and they can be referred by the following studies:

This study has introduced business model and discussed the commercial capital regarded as industrialization way, and it also transfers the basic study achievements related with intangible heritage to various digital service. Then, it integrates the network communication media and material antiques exhibition stand to establish a cycle industrialization mechanism that combines "public welfare" and "commerce" that mutual influence harmoniously. However, the study on industrialization mainly focuses on the introduction of commercial capital and the integration of current mature media, and more studies are needed on the practical meanings. Second, the following study on industrialization may focus on establishing relevant industrialization institution system and evaluation system on the exhibition content and so on.

It is believed that with further improvement of the wireless network bandwidth, the popularity of intelligent mobile terminal and information household appliances and the precision of video identification technique, the digital exhibition mode of intangible heritage of museum will draw more attention and be applied more widely. Finally, online database can be established which includes the entire digital intangible heritage, and it can provide a healthy and fresh existing method for intangible heritage. On the other hand, users will not be limited in the museum, and they can enjoy the service experiencing culture carried by the content of intangible heritage and related digital products anytime and anywhere.

\section{ACKNOWLEDGMENT}

This work was supported in Fudan University libe ral arts scientific research plan "JINMIAO" project, N O.08JM037.
House of Electronics Industry, pp.23-47, 2006.

[7] Shijin Xu and Hongjing Chen ect. Introduction to Digital Museum Shanghai scientific \& Technical Publishers, pp.3-30, 2007.

[8] Wen Zhang Introduction of non-material cultural heritage 1th Culture and Art Publishing House, pp.6-47, 2006.

[9] China Intangible Cultural Heritage Survey Manual 1th Culture and Art Publishing House, pp.15-39, 2007.

[10] Yue Qi and Xukun Shen Museum management and exhibition of digital resources Shanghai scientific \& Technical Publishers, pp.32-48, 2008.

[11] Bing wu Cao and Wen chang Li Museum of observation - the museum display of publicity and social service research Xue Yuan Publishing House, pp.2-19, 2005.

[12] Gang Chen New Media and Advertising China Light Industry Press, pp.12-38, 2002.

[13] Guang chao Feng Digital TV Advertising Communication University of China Press, pp.14-28, 2004.

[14] Nicholas Negroponte Being Digital Hainan Press, pp.1-54, 1996

[15] Lev Manovich, The Language of New Media, pp.3-130, 2004.

[16] Ben Shneiderman \& Catherine Plaisant, Designing the User Interface :Strategies for Effective Human-Computer Interaction, pp.110-128, 2004.



Bai, Jian-Song Born in Hebei of China Fudan University Department of Art and Design Lecturer research areas: multimedia design, interactive design, virtual reality design E-mail: baijiansong6@fudan.edu.cn

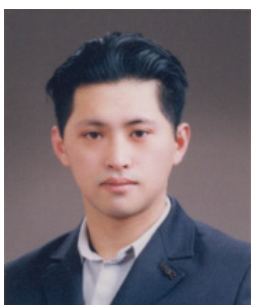

Boo, Jong-Chul Born in Jeju of Korea Digital Image Design Innovation Center, DongSeo University Department of Multimedia Design, Shanghai University of Engineering Science research areas: multimedia design, interactive design, virtual reality design

E-mail: asiavr@hotmail.com

\section{REFERENCES}

[1] Yanxiang Zhang, New Mediaart 1th Science Press, pp.3-30, 2005.

[2] Grau, Oliver and Ling Chen Virtual Art 1th Tsinghua University Press, pp.50-60, 2007.

[3] Mingquan Zhou Research by Digital Protection of Cultural Heritage BeiJing Normal University Publishing Group, pp.6-30, 2006.

[4] Alan Dix, Janet Finlay, Gregory D.Abowd Human Computer Interaction Publishing House of Electronics Industry, pp.5-50, 2006.

[5] Dan O'Sullivan, Tom Igoe Physical Computing 1th Tsinghua University Press, pp.13-35, 2006.

[6] Doug A.Bowman, Ernst Kruijff $3 D$ User Interface Publishing 